it would be tried as thrombolytic therapy in the future with anticoagulant.

\section{REFERENCES}

1) Sakuragawa, N., Takahashi, K. and Matsuoka, M.: Thrombolytic therapy using urokinase.
Acta Med. Biol. (Niigata) 25 : 125-135, 1978.

2) Sakuragawa, N. and Takahashi, K.: Thrombolytic therapy using urokinase : its mechanism and clinical use. Jap. J. Glin. Haemat. 18 : 726-731, 1977.

\title{
(3) Cerebrovascular Diseases in Young Adults
}

\author{
Sadatomo SHimojo, MD \\ The Second Department of Medicine, Jikei University School of Medicine, Tokyo
}

The incidence of cerebrovascular diseases (CVD) among young adults has been reported to range from 2 to $35 \%$ of those in all the age groups. It differs from those in higher age groups in that there are less incidence of hypertension, diabetes mellites, disorders of lipid metabolism and cerebral arteriosclerosis, and furthermore the underlying diseases are more varied. In Japan patterns of CVD is considered gradually shift!ng to those of western countries, namely occlusive GVD predominant due mainly to a recent increase in elderly populations. Despite of such a trend in this country, GVD as yet takes the first place for the cause of death and the ratio of cerebral hemorrhage to ischemic GVD still remains considerably high.

The purpose of the present study is to search whether such a trend may also be present in CVD of young adults under 39 years of age and further to elucidate pertinent underlying diseases or any specific etiologies in this age group.

Materials and methods: By reviewing hospital records 73 patient with various GVD under 39 years of age were selected from 686 GVD patients of all the age groups. Autopsy were performed in 123 cases of over all GVD in whom 18 were under 39 years of age. In GVD of young adults 42 were males and 31 females. For verification of diagnosis, cerebral angiography, RI scintigraphy, EEG, lumber puncture were performed in the majority of patients, particularly in those after 1976 added were computerized tomography (AGTA scan).

Results: (1) In younger age group the incidence of cerebral hemorrhage $(\mathrm{CH})$, cerebral infarct (CI), SAH and cerebral embolism (CE) were 27.4\%, 26.0\%, 26.0\% and $8.2 \%$ respectively. (2) The ratio GI v.s $\mathrm{CH}$ of young adults as compared in the decades, namely 1958 to 1967, 1968 to 1978 changed from 0.75 to 1.11 while the ratio increased from 1.46 to 3.59 in those above 40 years of age. The fact indicates that the increment of ratio is due to increased populations of the aged, which have occurred in the last decade. In younger age group pertinent underlying diseases as viewed from their past history were as the followings: hypertension in $18.8 \%$, renal diseases in $12.5 \%$, diabetes mellites in $3.1 \%$ respectively. It is noteworthy that 26 cases $(40.6 \%)$ proved to have no known underlying diseases. (4) Clinical symptomatology at the onset of GVD were as the followings: In $\mathrm{CH}$ disturbed level of consciousness, meningeal signs and pupillary abnormalities were frequently observed suggestive of a grave prognosis. In CI on the contrary disturbed 
level of consciousness were relatively infrequent and grade of hemiparesis were mild with a fair recovery as a rule. (5) Twenty-three of 73 cases $(27.3 \%)$ died of GVD as a direct cause of death among whom mortality of $\mathrm{CH}$ was $70 \%$ which was significantly higher than that of CI which was only $5.9 \%$. (6) In the present series included were those who manifested apoplectic insults in the course of SLE. Among 67 cases of SLE, 5 developed cerebrovascular accidents (7.5\%). All were females under 42 years of age, and the youngest 23. There were no relationship between the duration of SLE and cerebrovascular accidents. Though obscure as the etiologies of CVA 4 of 5 patients had hypertension particularly of diastolic pressure and all manifested an evidence of renal damage such as proteinuria or reduced

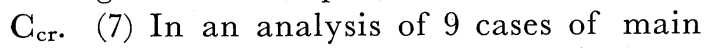
truncal artery occlusion in younger age group etiologies of occlusion were proved to be Moyamoya disease in 4, aortic arch syndrome in 2, atherosclerosis in 1 and unkown in 2. Sites of occlusion were predominantly in internal carotid artery (7 of 9 cases) followed by middle cerebral artery (1) and basilar artery (1). A case of lockedin syndrome and a subclavian steal syndrome associated with middle cerebral artery occlusion were included in this series. The etiologies of these two cases were not exactly known but arteritis could be well suspected.

Concluding remarks: Seventy-three patients under 39 years of age were found among 686 GVD (10.6\%) admitted in the period of 1958 to 1978 . The incidence was in fair agreement with $14.6 \%$ in autopsy materials of 123 cases. In this age group $\mathrm{CH}$ was found to be most frequent with varied underlying diseases such as hypertension, blood disorders, renal diseases and collagen diseases. For this reason the mortality of $\mathrm{CH}$ in this age group proved to be exceedingly higher than that of GVD above 40 years of age. CI v.s $\mathrm{CH}$ ratio as compared in two decade was uninfluenced in GVD of young adults while there was a sharp rise of ratio in those above 40 years of age in accord with general trend of increment in the nation wide surveys. On the contrary mortality of occlusive GVD in young adults was low with a fair functional recovery as a rule. There were varieties of etiologies for the occlusion of cerebral vessels including Moyomoya diseases, aortic arch syndrome, arteritis and so on but a considerable numbers manifested no known etiologies. In main truncal artery occlusion of cerebral vessels atherosclerosis was considered relatively rare and the site of prediction was mostly in internal carotid artery to differ from those above 40 years of age in whom occlusion of middle cerebral artery was most frequent with atherosclerotic origin.

\title{
(4) Diagnostic Problems of Acute Cerebrovascular Stroke
}

\author{
Yoshiaki TAZAKI, MD \\ Department of Internal Medicine, School of Medicine, Kitasato University.
}

The problems related to the diagnosis of acute cerebrovascular stroke have been investigated in the patients who were admitted into the Kitasato University
Hospital.

1. Ghronic subdural hematoma in the old age 